\title{
A New Modular Control Board for Pulse-Jet Cleaning of Dust Collector Filter Bags
}

\author{
Lorenzo Carosso \\ Department of Electronics \\ and Telecommunications, \\ Politecnico di Torino, Italy \\ lorenzo.carosso@polito.it
}

\author{
Daniel Gaiki \\ Department of Electronics \\ and Telecommunications, \\ Politecnico di Torino, Italy \\ daniel.gki@gmail.com
}

\author{
Silvano Bertoldo \\ Department of Electronics \\ and Telecommunications, \\ Politecnico di Torino, Italy \\ silvano.bertoldo@polito.it
}

\author{
Marco Allegretti \\ Department of Electronics \\ and Telecommunications, \\ Politecnico di Torino, Italy \\ marco.allegretti@polito.it
}

\begin{abstract}
This work presents a timing system modular control board to be used for pulse-jet cleaning of dust collector filter bags or pneumatic conveying systems. Many systems of this kind are already available on the market but only some of them have the ability to operate in full range alternating current power supplies. The presented versatile and innovative control board can control the electro valves, either the ones operating with alternating current and those in direct current (since they are both used in the industrial plants). For the same reason, the designed prototype system can be powered with different voltages, widening the number of potential industrial applications. The electronic board has as main advantage the ability to fully automate the cleaning cycle. It can drive many electro valves simultaneously since it is possible to connect more control boards together if needed. It also allows the user to separate the control section of the system from the section dedicated to the actuation of the electro valves. The electro valves can be controlled from a PC terminal via ethernet. The work describes the adopted solutions and the steps made for the design of the entire prototypal modular board, with particular focus on the innovative timing system.
\end{abstract}

Keywords-pulse jet baghouse; pulse jet electro valve; pulse jet cleaning; electro valve actuator; remote monitoring bag house; $A C$ electro valves control; DC electro valves control; prototypal timing system

\section{INTRODUCTION}

Surface filter systems are heavily applied in air filtration processes for industrial applications and the use of a bag (filter media) cleaning subsystem by using compressed air jets is widespread as well. The pulse-jet type of dust filter was invented and developed and introduced on the market in the late 1950's [1]. The basic device patent has expired and there are now many manufacturers of pulse-jet collector type $\lceil 2\rceil$. Core business of industries using Pulse-Jet Fabric Filtration (PJFF) regard power generation, incineration, chemical, steel, cement, food, pharmaceuticals, metal working, aggregates and carbon black [3]. The research group of remote sensing and innovative sensors development of The Department of Electronics and Telecommunications (DET) of Politecnico di Torino, has a great interest in designing new prototypes and new solutions for both academic research purposes and industrial innovative applications. Adopted solutions were always realized considering keeping the cost as low as possible for industrial applications. They cover a large range of applications highlighting the multidisciplinary attitude and interests of the research group. During the year some environmental monitoring sensors have been developed such as a microwave radar for rain monitoring [4], sensors and techniques for forest fire monitoring $[5,6]$ etc. Starting from the first multipurpose board realized in 2012 [7], a large number of industrial projects have also been realized. They include a new anti-theft alarm systems for photovoltaic panels [8], a wireless sensor network (WSN) for smart gas metering (which was also documented by the Italian patent for utility model No. 0000279556) and more recently RFID tags and readers to be installed on unmanned aerial vehicles (UAVs) $\lceil 9\rceil$ and sensors for health structure monitoring [10]. Exploiting the high expertise of the research group in of sensor board realization, this work presents the realization of a new low cost and versatile solution for modular control board for pulse-jet cleaning of dust collector filter bags.

Considering the energy costs involved in pressurizing air to clean the filter media, the proper design of an innovative adhoc cleaning system is of high interest. A widely used system for implementing an automatic cleaning of an air-jet dust collector consists of a differential pressure gauge and a programmable controller. The differential pressure gauge measures the pressure difference between the dirty air side and the clean air side of the bag filters in the baghouse: our system is based on this consideration. Clean filter systems are called "reverse pulse jet". In the filter media cleaning subsystem, high pressure gas injections and short duration are used in the reverse direction of the pressure gradient and cause a loosening of the dust layer from the surface of the bag: the dust layer is then collected in the dust tank located at the bottom of the filter housing (see Figure 1). Such systems work in countercurrent by compressed air jets controlled by solenoid valves, which in turn are activated according to a predefined program [11]. The impulse type and the pause time between two consecutive pulses are programmed with the sequencer, according to a predefined and not modifiable structure. This procedure causes an excessive wear of the sleeve of the filter. The operating principle of the sequencers available on the market is the following: first, the cleaning cycle is activated. The sequencer 
detects the first available electro valve and, on the basis of the pre-set working time, the electro valve is opened to recall a specific amount of compressed air required to clean the filter until the completion of a cleaning cycle. Then, after a pause interval, the sequencer automatically recalls and opens the following available electro valve to start a new cleaning cycle.

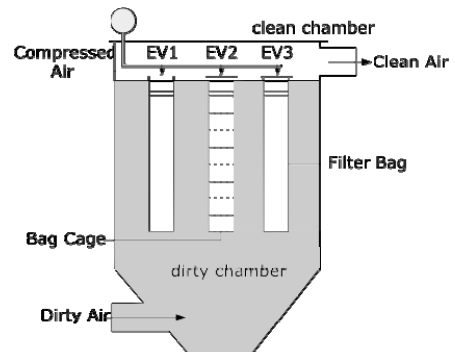

Fig. 1. Lateral view of bag house chamber.

Therefore, it is not possible to directly check the correct working of the cleaning system or the degree of cleanliness achieved by the filter when a single cleaning cycle is completed (i.e. when a single electrovalve has been activated). Since it is not possible to interact directly with the sequencer, the control procedure is performed usually from time to time by means of external instrumentation. Based on the obtained results, it is then possible to re-calibrate the time necessary for carrying out a whole cleaning cycle. Evidently, the described technique has a set of undesired effects, the most important of them being:

- The lack of a real time control of the degree of cleanliness achieved by the air/gas/steam that passes through the single filter. This limitation could be dangerous in the case of treatment of dangerous substances.

- The need of a qualified operator to work on the sequencer (even remotely) to adapt the system to the new conditions found during the subsequent checks performed with external instrumentation.

- The impossibility to obtain real time reports during the filters' working operations.

- The impossibility to understand in real time the potential causes of malfunctioning. Therefore, it is not possible to intervene promptly to avoid more filter damage and even to the complete industrial process.

The innovation implemented here concerns a new modular control unit to optimize the cleaning process. Specifically, such unit performs multiple tasks:

- Managing the plurality of voltages input (including AC or DC) from the solenoid valves, thus allowing system adaptation to different industrial processes.

- Performing the automation of the cleaning cycle. The automatic re-definition of the pulse and pause interval can be obtained according to the cleaning results achieved and measured inside the filters.

- The possibility of operation with either fixed or variable cleaning cycle. It is possible to set the "cleaning cycle" operational mode according to the pressure difference between filter input and output (see section II).

- The possibility to correlate the generated pulse and the pause interval to the residual capacity in compressors that are supplying air jets. Air shots too long and close to each other air shots can drastically decrease the pressure in input.

\section{FUNCTIONAL LOGIC DESCRIPTION OF THE SYSTEM}

The operations performed by the prototypal system presented in this work can be better illustrated using the scheme represented in Figure 2. The baghouse is divided horizontally in four sectors and each one contains four electro valves, represented by the EV\# codes, and vertically in two sections: the clean chamber, where the cleaned air comes out of the filters, and the dirty chamber, where the air is introduced to be cleaned. The electro valves are responsible to control a specific air pulse jet for a group of bags that clean the dirty air through the bag cages.

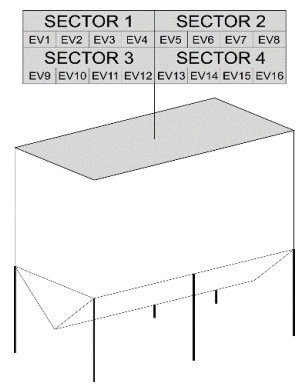

Fig. 2. Baghouse division on 4 sectors. Each sector contains 4 electrovalves.

Depending on the size of the filter, the number of sections and valves may vary. Only one electro valve at a time is excited, the wash cycle is completed when all the valves are energized progressively. With this prototypal system, a large set of programmed sequences of electro valve activations is also possible. However, a good rule is to avoid to fire electrovalves placed consecutively. Since each cleaning cycle causes a reduction of the filter lifetime, to avoid excessive wear on the filter elements, the washing system must be optimized to act only when necessary. In that case, the electro valve shooting time and the interval between a shot and the next may vary depending on the values measured by the pressure sensor inside the filter chamber. Usually, for a single electro valve the time to shoot can vary from $80 \mathrm{~ms}$ to $140 \mathrm{~ms}$. The time interval between two consecutive shots of the same valve may be defined in two ways and depends on whether it chooses a "fixed cycle" or "variable cycle" operational mode, described below. Both cycle types can be programmed with the boards realized in this project. Evidently, both the shot duration and the interval period must be proportional to the capacity of compressors that provide compressed air: shots too long and close can drastically decrease the air pressure in input, reducing the quality of filtering operation.

\section{A. Fixed Cycle Operational Mode}

The "fixed cycle" operational mode sets the number of cycles to be run during a specific time interval. Let us assume, 
for instance, that a number of 3 washing cycles is needed during one hour. It means that all 16 valves (if the bag filter is made by a set of 16 electro valves) must shoot 3 times in one hour. As a result, the system will calculate the time interval between 2 consecutive shoots as reported in (1):

$$
t_{\text {interval }}=\frac{3600-\left(t_{\text {shot }} \cdot n_{\text {valve }} \cdot n_{\text {cycle } / \text { hour }}\right)}{n_{\text {valve }} \cdot n_{\text {cycle } / \text { hour }}}
$$

where: $t_{\text {interval }}[\mathrm{s}]$ is the time interval between two consecutive wash cycles, $t_{\text {shot }}\lceil\mathrm{s}\rceil$ the time interval between electro valve drives, $n_{\text {valve }}$ the total number of valves and $n_{\text {cycle/hour }}$ [cycle/hour] the total number of cycles in one hour.

\section{B. Variable Cycle Operational Mode}

With the "variable cycle" operational mode, the shooting range time depends on the difference in pressure (DP) between the "clean chamber" and the "dirty chamber" of the filter, measured through a differential pressure sensor. At first it is necessary to determine the desired DP to be maintained and this value is used as a set-point to trigger a new cleaning cycle. With this information, the system automatically evaluates the time interval between two consecutive shots. Of course, if DP is greater than the set-point, the system increases the washing frequency, thus decreasing the time between two consecutive shots. Otherwise, if the DP is lower than the set-point, the time between two shots needs to be increased. At specific intervals (usually 30-100 seconds) the system controls the DP and compares it to the set-point; if it is greater than a preset threshold (called "dead band"), the system changes the interval time at a fixed percentage $(1 \%-10 \%)$, otherwise it stays unchanged. If the DP is very low and the filter already has a very long interval time (usually limit values are set a priori), the washing cycle can be stopped. It is resumed when DP is greater than a certain threshold.

\section{General Notes}

Usually, the factory set of the pulse frequency can range from a few seconds to a few minutes; this clearly depends on the number of valves and the filter size. The number of valves inside a filter can vary from 10-16 up to $180-200$. If the filter is stopped for low DP or low compressed air by the compressors, when it is resumed it must restart from the valve next to the one where it was previously stopped. In this way, all the sleeves of a filter are washed the same number of times. Specific sensors can measure the cleaning results inside the filter and according to the programmed settings, a potential re-definition of the pulse and pause interval between consecutive pulses can be automatically obtained.

\section{ELECTRONIC BLOCKS DESCRIPTION}

The system is made by a set of different electronic blocks. Their description and their functionalities are presented below, according to the different subsystems of the modular control board for pulse-jet cleaning of dust collector filter bags:

- power supply

- human machine interface

- output and input of the controller board (the subsystem that supervises the whole modular system and can be programmed to automatically re-configure the operational model)

- output of the electro valves driver board (the board that directly controls the electro valves activation which is directly linked with the controller board).

A scheme of the connection between control board and electro valve output control boards using a serial interface is shown in Figure 3.

\section{A. Power Supply}

Industrial pulse jet timer controllers (PJTCs), commonly used for baghouse filters, established that control boards that use a universal alternating current (AC) input in a full range power supply $115 / 230 \mathrm{VAC}$. These control boards usually have on-board power supplies. It means that whenever there is a problem with the circuit power supply, the entire control board must be replaced and the new control board must be properly reprogrammed. The developed solution aims to separate the different possible problems and to make the solution more flexible and versatile. Together with common full AC range power supply, a system that has a $24 \mathrm{~V} \mathrm{DC}$ power supply is included. It adds robustness to the system and, if failure occurs, the power supply can easily be substituted without need of reprogramming the entire system, giving thus a noteworthy advantage in terms of cost, materials and time saving.

\section{B. Human-Machine Interface}

A large part of timer controller has an interface that operates fully integrated on board without the possibility of sending or receiving data from a remote management system. For instance the cement industry routinely implements human machine interface (HMI) and supervisory control and data acquisition (SCADA) interfaces for a wide range of processes, maintenance and environmental compliance requirements. These sophisticated systems do not offer the possibility to adapt to specialized systems dedicated to particulate matter (PM) control and associated with advanced baghouse diagnostics. PM and baghouse specific systems are certified with fieldbus ports to communicate with existing PLCs and DCS systems to enable seamless integration with most of the existing plant equipment. A common and versatile family of standards and technologies that can be used in innovative electronic boards is the industrial Ethernet (IE). It consists on the use of Ethernet technologies in an industrial environment with protocols that provide determinism and real- time control (e.g. EtherCAT, EtherNet/IP, PROFINET, POWERLINK, SERCOS III, CC-Link IE, and Modbus/TCP [12, 13]). Using IE, data collected on the filter bag can be stored and displayed on a dashboard, and by sharing them on a shared platform it is possible to compare them with others collected by other systems. Moreover, using devices connected to a network and a centralized interface capable to get data and send commands to all devices, it is possible to control the electro valves using different information acquired by other sensors distributed on the industrial plant. In order to overcome the above difficulties, the proposed control board is equipped with an IE controller, thus giving the board the whole set of described advantages, including the possibility to remotely reprogramming the systems, if needed. 


\section{Outputs of the Controller Board}

The control systems available on the market to integrate everything on the same board present several inputs and outputs. It is possible to find boards with $8 / 16 / 32$ outputs to control solenoid valves. This could be a problem when having in mind to further expand the system. If it is necessary to control more electro valves and if the system has a limited number of outputs, it should be replaced and reprogrammed, leading to extra working time and adjustments. The proposed electronic board is actually a modular system. It is possible to add more output modules to the solenoid valves as needed, without changing the control board, by simply adding new expansion boards in which the actuation drivers are placed. This modularization allows the separation of the actuation modules (also referred to as electro valve driver board) from the control module. When a failure in one of the actuation modules occurs, any of the actuation modules may be substituted without modifying the setup and configurations in the control module. The board control that drives the solenoid valves is controlled by a serial interface. Even if the serial communication interface is not a recent one, it allows increasing the number of outputs without compromising many pins of the microcontroller and, at the same time, giving robustness to the system. The serial communication used is then a "synchronous serial communication" where each actuating board of the solenoid valve has a shift register circuit that can trigger sequentially each valve. Each shift register circuit has 8 outputs that are used for the control of the electric valves. It is possible to increase the number of outputs by simply linking several registers together. Other outputs of the control board are the alarm signals. They can be programmed according to a predefined setup or depending on specific inputs coming from dedicated sensors aiming to trigger the alarm signals. These outputs can be used to give a visual feedback on the system-operating situation or to communicate the presence of a possible problem detected by an external sensor. The alarm output is an optically isolated transistor that can trigger an external device (e.g. acoustic or visual alarm signaling devices).

\section{Input Sensors of the Controller Board}

A DP sensor is commonly used as input of the timing control board. The developed system is engineered to allow both the input of the DP sensors and the input of a second control based on another sensor that can be chosen according to specific needs of the industrial process. The automation of the system is made according to the input of all those sensors.

\section{E. Outputs of the Electro Valve Driver Board}

Due to the existence of different types of electronic valves in the market, a board with a power interface operating in both $\mathrm{DC}$ and $\mathrm{AC}$ has been designed, thus increasing the system's versatility. Another possibility given by the present modular system is the use of AC or DC solenoids using the same circuit. Considering a large set of electro valves available on the market, it is possible to find electro valves with voltages that range in the interval of $115 \mathrm{~V}-230 \mathrm{~V} \mathrm{AC}$ and $24 \mathrm{~V} \mathrm{DC}$, which are generally widespread in the industry. Two common types of control boards for electro valves are commercially available. They are different on the basis of the circuit used as actuator for the solenoid valves. Generally the control boards that control AC electro valves use relay or TRIAC (TRIode for Alternating Current). The disadvantage of the circuits using TRIAC is that they work only for AC thus losing the probability to control DC electro valves. In this work, a circuit is proposed that can operate with both $\mathrm{AC}$ and $\mathrm{DC}$ valves. It uses a bidirectional electronic switch based on MOSFETS (a type of circuit widely used in solid-state relays). By using this topology is possible to design a robust circuit at low cost (very similar to a simple and standard circuit based on common relays). Figure 4 illustrates two bi-directional switches used to control two different electrovalves separately. In this case the signals defined by IN1 and IN2 are connected in phase with respect to the signal considering $\mathrm{AC}$ or at the positive signal in case of DC. The outputs defined by Pout1 and Pout2 are connected to the returns that feed the solenoid valves. This circuit has the possibility to use both AC and DC without the need to change the power supply modules. Through the Shunt resistors (represented in Figure 4 as Shunt 1-4) the electric current that supplies the electro valves can be monitored, thus controlling if some valve is disconnected or damaged. If a short circuit occurs, the activation of a specific electro valve may be canceled and in this case an alarm signal can be generated as output of the modular control board and sent to the system that controls the industrial plant exploiting the IE communication.

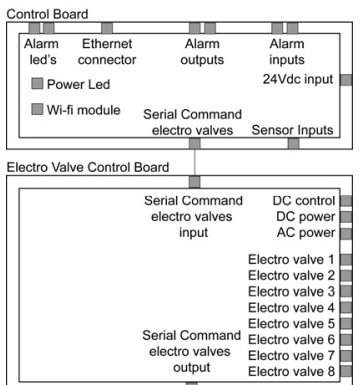

Fig. 3. Connection between control board and electro valve output control boards using a serial interface

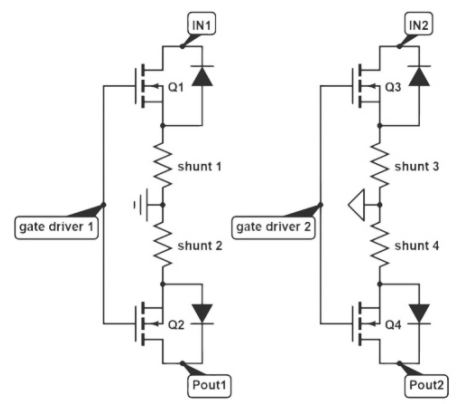

Fig. 4. Bidirectional switches used to control two separated electro valves.

\section{INSTALLATION}

The proposed modular electronic board can be integrated with a large number of systems commonly and widely present in a large number of industrial plants. Generally, other systems must be installed together with the air filtration system and they already have their own power supplies. On the diagram represented in Figure 5 it is possible to note the presence of two 
different circuits: the proper control board of the entire system and the board devoted to electro valve controlling (electro valve driver board). It is evident that supplying the circuits directly with the $24 \mathrm{~V}$ source, if available, could be optional or to supply them with the standard AC provided by the power grid. At the same time, it is possible to activate electro valves of both types, as described above. Figure 6 represents an example of realization of a modular installation using the realized boards. The smaller board on top is the control board (Figure 7), the other three are the electro valve driver boards (Figure 8). It is evident that multiple control boards can be interconnected to each other.

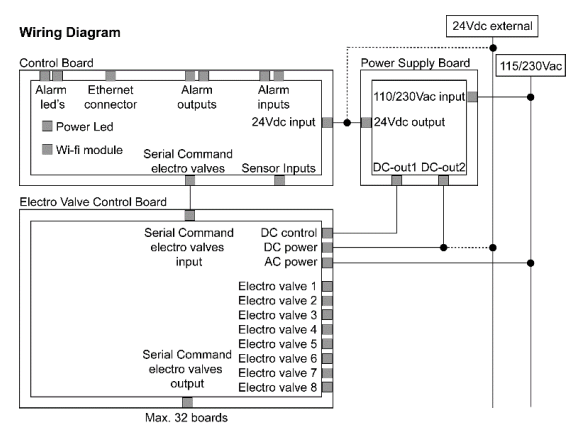

Fig. 5. Wiring diagram of the installation of the boards on the system.

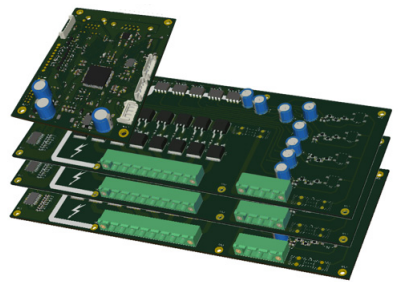

Fig. 6. Example of assembled system with 1 controller board and 3 electrovalves driver boards.

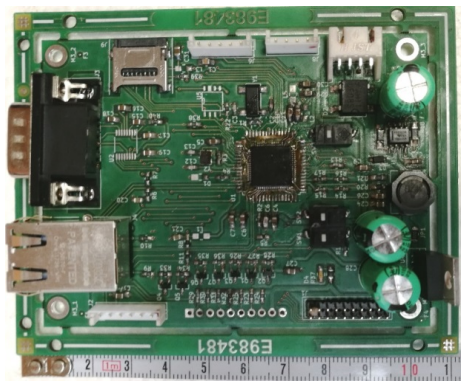

Fig. 7. The realized control board.

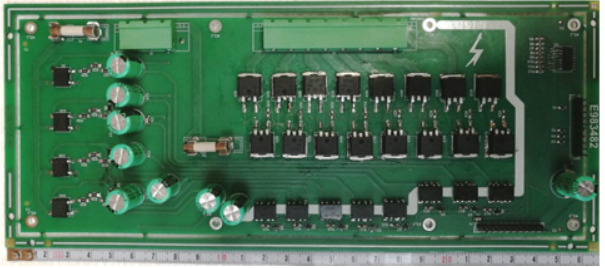

Fig. 8. Picture of the realized driver board.

\section{CONCLUSION}

Present work presents the prototypal realization of a modular low cost control board for a system for baghouses cleaning. Some advantages have been already described in the introduction. However, the improvements introduced by these boards compared to the usual practice are mainly related to their possible integration with already existing commercial systems and to the versatility of the system itself. The same board allows the automation of the cleaning cycle according to measured parameters acquired by dedicated sensors and the possibility of operation with both fixed and variable cleaning cycles. The modular structure of the boards allows users to install them in any desired number without difficult operations of reprogramming the entire systems. The possibility to connect different drive boards together, controlled by a single control board, allows a wide number of drive outputs, widening the number of potential applications. The possibility to automatically reprogram the entire system using the information provided by sensors devoted to check the state and the "health" of the filter to be cleaned is very significant. The configuration and the programming of the control board can be easily made by using a common PC. It is possible to setup the number of valves to be activated and the system can be configure remotely, since the board is equipped with an IE that allows the system itself to be accessible within a company network and even in the world of internet (of course, only for authorized users). For what concerns the electric drive, it is important to highlight that the proposed prototypal board allows using the same drive circuit for different types of electro valves, thanks to its high versatility.

\section{REFERENCES}

[1] R. E. Frey,T. V. Reinauer, "New filter rate guide", Air Engineering, Vol. 30, 1964

[2] E. Bakke, "Optimizing Filtration Parameters", Journal of the Air Pollution Control Association, Vol. 24, No. 12, pp. 1150-1154, 1974

[3] A. K. Choudhary, A. Mukhopadhyay, "Particulates: Selection of cleaning pulse pressure for pulse jet fabric filtration", Filtration + Separation, Vol. 50, No. 4, pp. 28-30, 2013

[4] M. Gabella, R. Notarpietro, S. Bertoldo, A. Prato, C. Lucianaz, O. Rorato, M. Allegretti, G. Perona, "A Network of Portable, LowCost, X-Band Radars”, in: Doppler Radar Observations - Weather Radar, Wind Profiler, Ionospheric Radar, and Other Advanced Applications, InTech, 2012

[5] A. Losso, L. Corgnati, S. Bertoldo, M. Allegretti, R. Notarpietro, G. Perona, "SIRIO: an integrated forest fires monitoring, detection and decision support system using low cost commercial sensors. Performance and result of the experimental installation in Sanremo (Italy)", in: C. A. Brebbia (ed.), Modelling, Monitoring and Management of Forest Fires III, pp. 79-90, WIT Press, 2012

[6] A. A. Khamukhin, A. Y. Demin, D. M. Sonkin, S. Bertoldo, G. Perona, V. Kretova, "An algorithm of the wildfire classification by its acoustic emission spectrum using Wireless Sensor Networks", Journal Of Physics: Conference Series, Vol. 803, No. 1, Article No. 012067, 2017

[7] O. Rorato, C. Lucianaz, S. Bertoldo, M. Allegretti, G. Perona, "A multipurpose node for low cost wireless sensor network", IEEE APWC, Cape Town, South Africa, pp. 247-250, September 2-7, 2012

[8] S. Bertoldo, O. Rorato, C. Lucianaz, M. Allegretti, "A Wireless Sensor Network ad-hoc designed as anti-theft alarm system for 
photovoltaic panels", Wireless Sensor Network, Vol. 4, No. 4, pp. 107-112, 2012

[9] G. Greco, C. Lucianaz, S. Bertoldo, M. Allegretti, “A solution for monitoring operations in harsh environment: a RFID reader for small UAV”, IEEE ICEAA, Turin, Italy, pp. 859-862, September 7-11, 2015

[10] L. Carosso, M. Allegretti, S. Bertoldo, "Innovative Wireless Sensor Network for monitoring and diagnosis of civil infrastructures", IEEE APWC, Verona, Italy, pp. 8-11, September $11-15,2017$

[11] P. S. Abhishek, P. N. Ramachandran, "Design of pleated bag filter system for particulate emission control in cement industry", International Research Journal of Engineering and Technology, Vol. 2 No. 05 , pp. 763-768, 2015

[12] Z. Lin, S. Pearson, An inside look at industrial Ethernet communication protocols, Texas Instruments, 2017

[13] R. Zurawski (ed.), Industrial Communication Technology Handbook, CRC Press, 2014 